\title{
VLT/MUSE illuminates possible channels for Lyman continuum escape in the halo of SBS 0335-52E ${ }^{\star}$
}

\author{
E. C. Herenz ${ }^{1}$, M. Hayes ${ }^{1}$, P. Papaderos ${ }^{2}$, J. M. Cannon ${ }^{3}$, A. Bik ${ }^{1}$, J. Melinder ${ }^{1}$, and G. Östlin ${ }^{1}$ \\ 1 Department of Astronomy, Stockholm University, AlbaNova University Centre, 10691 Stockholm, Sweden \\ e-mail: christian.herenz@astro.su.se \\ 2 Instituto de Astrofísica e Ciências do Espaço - Centro de Astrofísica da Universidade do Porto, Rua das Estrelas, 4150-762 Porto, \\ Portugal \\ 3 Department of Physics \& Astronomy, Macalester College, 1600 Grand Avenue, Saint Paul, MN 55105, USA
}

Received 21 August 2017 / Accepted 2 October 2017

\begin{abstract}
We report on the discovery of ionised gas filaments in the circum-galactic halo of the extremely metal-poor compact starburst SBS 0335-052E in a $1.5 \mathrm{~h}$ integration with the MUSE integral-field spectrograph. We detect these features in $\mathrm{H} \alpha$ and [O III] emission down to a limiting surface-brightness of $5 \times 10^{-19} \mathrm{erg} \mathrm{s}^{-1} \mathrm{~cm}^{-2} \operatorname{arcsec}^{-2}$. The filaments have projected diameters of $2.1 \mathrm{kpc}$ and extend more than $9 \mathrm{kpc}$ to the north and north-west from the main stellar body. We also detect extended nebular He II $\lambda 4686$ emission that brightens towards the north-west at the rim of a starburst driven super-shell. We also present a velocity field of the ionised gas. The filaments appear to connect seamlessly in velocity space to the kinematical disturbances caused by the shell. Similar to high- $z$ starforming galaxies, the ionised gas in this galaxy is dispersion dominated. We argue that the filaments were created via feedback from the starburst and that these ionised structures in the halo may act as escape channels for Lyman continuum radiation in this gas-rich system.
\end{abstract}

Key words. galaxies: starburst - galaxies: halos - galaxies: individual: SBS 0335-052E - techniques: imaging spectroscopy

\section{Introduction}

Pinpointing the sources that are relevant for reionising the universe at $z \gtrsim 7$ is one of the current major goals in observational cosmology. Hydrogen ionising photons $\left(E_{\gamma} \geq 1 \mathrm{Ry}\right)$ from low-mass star-forming galaxies are one viable candidate (e.g. Faisst 2016; Schaerer et al. 2016), but direct observations of these photons leaking from high- $z$ galaxies are scarce (e.g. Rutkowski et al. 2017). Moreover, the processes enabling the escape of this so-called Lyman continuum (LyC) radiation from the interstellar- and circum-galactic medium (CGM) are not well constrained. In principle feedback from supernovae can carve ionised gas cavities through the CGM, thereby creating channels through which the LyC can leak into the intergalactic medium (Fujita et al. 2003). Observational inferences of such processes have to be made from the neutral and ionisied gas phase in the CGM (Bik et al. 2015). At high redshifts, however, direct observations of the CGM in its neutral phase are currently impossible (Obreschkow et al. 2011) while directly detecting its ionised phase remains challenging (Rauch et al. 2016; Wisotzki et al. 2016; Finley et al. 2017). Fortunately, observations of nearby galaxies with properties comparable to systems at the highest redshifts provide a promising alternative for detailed examinations of the relevant physical processes in the early universe (for a recent review see Hayes 2015).

The SBS 0335-052 system at a redshift of $z=0.0135$ $(d=54 \mathrm{Mpc})$ consists of a pair of extremely metal-deficient star-forming dwarf galaxies separated by $22 \mathrm{kpc}$ (in projection), which have long been recognised as a special laboratory for such

\footnotetext{
* Based on observations collected at the European Organisation for Astronomical Research in the Southern Hemisphere under ESO programme 096.B-0690.
}

studies (Izotov et al. 1990). In fact, with $12+\log (\mathrm{O} / \mathrm{H}) \lesssim 7.15$ (3\% of the solar value) the western galaxy - SBS 0335-052W (discovered by Pustil'nik et al. 1997) - is one of the most metalpoor emission line galaxies known (Izotov et al. 2009). Observations of galaxies with such low oxygen abundances are rare and only a handful of objects comparable to SBS 0335052W are known (Guseva et al. 2017; Izotov et al. 2017). The brighter eastern galaxy - SBS 0335-052E - shows only a slightly higher nebular oxygen abundance, and thus also belongs to the elusive group of extremely metal-poor compact star-forming galaxies. Hubble Space Telescope (HST) observations of this galaxy reveal six young $(t<10-25 \mathrm{Myr})$ super-star clusters (SSCs; with masses $M_{\mathrm{SSC}}>10^{5} M_{\odot}$ and star formation (SF) rates $S F R_{\mathrm{SSC}} \lesssim 1 M_{\odot} \mathrm{yr}^{-1}$ ) that are within $\sim 500 \mathrm{pc}$ of each other (Thuan et al. 1997; Adamo et al. 2010). Observations of the $21 \mathrm{~cm}$ line revealed that the two galaxies are surrounded by a large HI complex (Pustilnik et al. 2001). This HI halo exhibits elongated tidal tails to the east and west as well as a faint diffuse bridge connecting both galaxies (Ekta et al. 2009). The inferred neutral hydrogen column density towards brightest starforming regions in SBS 0335-052E is $2 \times 10^{21} \mathrm{~cm}^{-2}$. Analysis of HST UV spectroscopy with GHRS (Thuan \& Izotov 1997) and COS (James et al. 2014) points at an even higher neutral column of 5-7 $\times 10^{21} \mathrm{~cm}^{-2}$. Thus, the optical depth for photons above $1 \mathrm{Ry}$ is $10^{4}$ or higher, implying total absorption of LyC radiation from the young stellar population in SBS 0335-052E along our sightline.

In this letter we report on a discovery of low surfacebrightness (SB) filamentary tails in $\mathrm{H} \alpha$ and [O III] emission that emanate to the north (N) and north-west (NW) of SBS 0335-052E. We speculate that these highly ionised tails may act as channels for LyC photon leakage. 

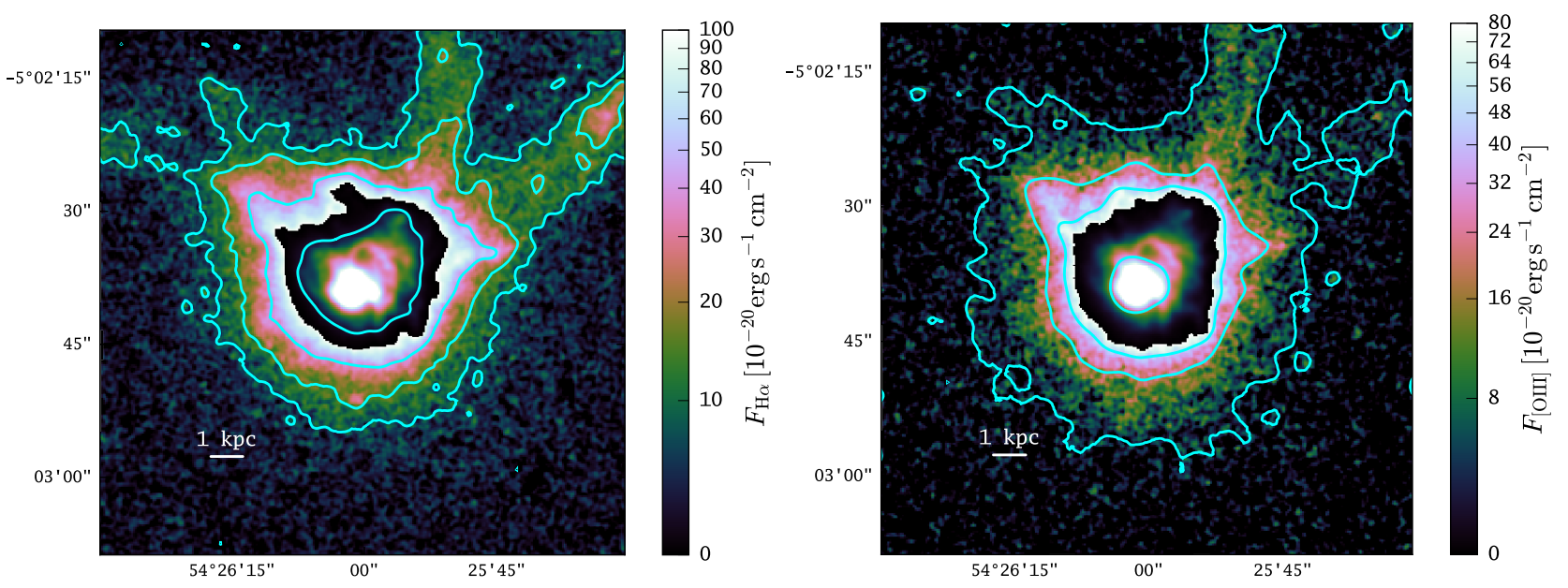

Fig. 1. $\mathrm{H} \alpha$ (left) and [O III] $\lambda 5007$ (right) narrowband images of SBS 0335-052E created from the MUSE data cube. East is left and north is up. The colour scale within the high-SB region encodes fluxes from $10^{-18}$ to $10^{-15} \mathrm{erg} \mathrm{s}^{-1} \mathrm{~cm}^{-2}$ for $\mathrm{H} \alpha$ and $8 \times 10^{-19}$ to $8 \times 10^{-16} \mathrm{erg} \mathrm{s}^{-1} \mathrm{~cm}^{-2}$ for [O III], while for the outer low surface-brightness region the encoding is shown by the colour bar on the right. An asinh scaling is used. SB contours are drawn at $[2.5,5,12.5,1250] \times 10^{-18} \mathrm{erg} \mathrm{s}^{-1} \mathrm{~cm}^{-2}$ arcsec ${ }^{-2}$ for $\mathrm{H} \alpha$ and $[0.5,5,12.5,1000] \times 10^{-18} \mathrm{erg} \mathrm{s}^{-1} \mathrm{~cm}^{-2} \operatorname{arcsec}^{-2}$ for [O III]. To highlight the low-SB features, the images were smoothed with a $\sigma=1 \mathrm{px}\left(0.2^{\prime \prime}\right)$ Gaussian kernel.

\section{Observations, data reduction, and creation of narrowband images}

VLT/MUSE (Bacon et al. 2010) observations of SBS 0335-052E were obtained in service mode under clear skies on November 16 and 17, 2015. The seeing varied between $0.9^{\prime \prime}$ and $1.2^{\prime \prime}$. We used the wide-field mode without the second-order blocking filter. The total exposure time was $5680 \mathrm{~s}(8 \times 710 \mathrm{~s}$ integrations). No separate sky exposures were taken. We reduced and calibrated the data using the MUSE data reduction system (DRS; Weilbacher et al. 2014) version 1.6.2. After reduction we were equipped with a sky-subtracted, wavelength, and flux calibrated data cube covering a field of view $(\mathrm{FoV})$ of $1^{\prime} \times 1^{\prime}$ over a spectral range from $4600-9370 \AA$. From this cube we produced an emission-line only data cube by subtracting an in spectral direction median-filtered (180 ̊ filter width) continuum-only cube (see Sect. 4.1 in Herenz \& Wisotzki 2017). We then synthesised $8 \AA$ wide narrowband images centred around the emission lines $\mathrm{H} \alpha$, [O III] $\lambda 5007$, and the He II $\lambda 4686$, thus probing zones ionised by $E_{\gamma} \geq 1 \mathrm{Ry}, E_{\gamma} \geq 2.58 \mathrm{Ry}$ and $E_{\gamma} \geq 4$ Ry photons, respectively. The final images are shown in Fig. 1 for $\mathrm{H} \alpha$ and [O III] (after applying the correction described below) and Fig. 2 for He II.

Owing to the large spatial extent of the $\mathrm{H} \alpha$ and [O III] emission the standard sky correction resulted in over subtraction of $\mathrm{H} \alpha$ and [O III] flux. This resulted in negative zero-point offsets in the $\mathrm{H} \alpha$ and [O III] narrowband images and, moreover, visible $\mathrm{H} \alpha$ and [O III] emission line features in the DRS calculated sky spectrum. After ensuring that no real skylines were present at the affected wavelengths, we removed those features from the calculated sky spectrum by linear interpolation over the bracketing wavelengths. Feeding this modified spectrum back into the DRS removed the over subtraction for $\mathrm{H} \alpha$ completely, while in the [O III] band a remaining small average offset of $2 \times 10^{-20} \mathrm{erg} \mathrm{s}^{-1} \mathrm{~cm}^{-2}$ had to be corrected manually.

\section{Results}

In the high $\mathrm{SB}$ region of our $\mathrm{H} \alpha$ and [O III] images (Fig. 1) we resolve in great detail the super-shell structure $(r \approx 1.3 \mathrm{kpc})$ first discussed in Thuan et al. (1997). Extending further to the $\mathrm{N}$ and NW from that shell two low-SB filaments are seen. In

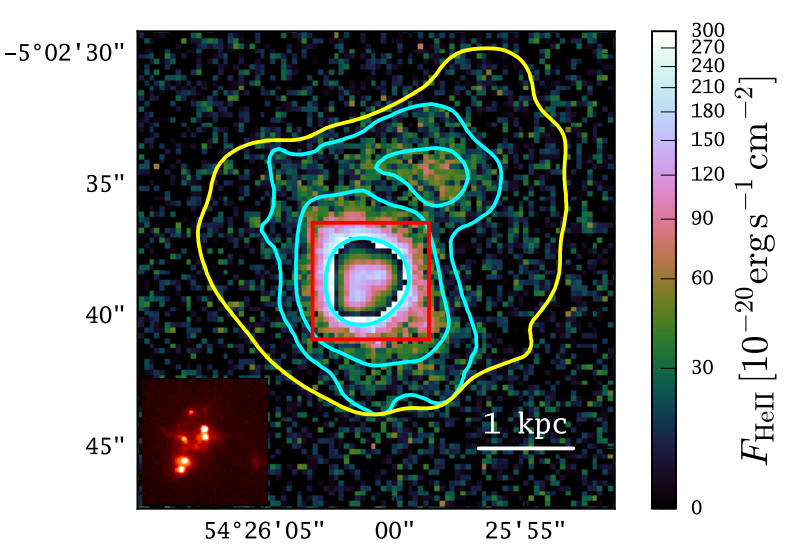

Fig. 2. He II narrowband image magnified on the central region. The yellow contour is the $1.25 \times 10^{-15} \mathrm{erg} \mathrm{s}^{-1} \mathrm{~cm}^{-2} \operatorname{arcsec}^{-2}$ contour from the $\mathrm{H} \alpha$ image (Fig. 1, left). Colour scale in the inner high-SB region extends from $3 \times 10^{-18}$ to $3 \times 10^{-17} \mathrm{erg} \mathrm{s}^{-1} \mathrm{~cm}^{-2} \operatorname{arcsec}^{-2}$, while for the outer region it is shown by the colour bar on the right. An asinh scaling is used. Contours correspond to $[2.5,100,1000] \times 10^{-18} \mathrm{erg} \mathrm{s}^{-1} \mathrm{~cm}^{-2} \operatorname{arcsec}^{-2}$. The inset in the bottom left shows the emsission line free HST WFC3/F550M image from the galaxy within the region indicated by the red square.

the north-east (NE) a third, less pronounced filament is also noticeable. A deep long-slit spectrum by Izotov et al. (2001) already indicated the presence of extended emission in the SE NW direction. Our new MUSE data is significantly deeper, detecting extended $\mathrm{H} \alpha$ as faint as $5 \times 10^{-19} \mathrm{erg} \mathrm{s}^{-1} \mathrm{~cm}^{-2} \operatorname{arcsec}^{-2}$. Moreover, the spatially resolved nature of our MUSE data allows us to map the filamentary morphology of the extended low-SB emission for the first time. The filaments appear to continue beyond the MUSE field of view (FoV), permitting us only to provide lower limits of their radial extend, i.e. $9.7 \mathrm{kpc}\left(35.1^{\prime \prime}\right)$ and $9.3 \mathrm{kpc}\left(33.7^{\prime \prime}\right)$ for the NW and N filament, respectively.

The filamentary "ears" differ in their $[\mathrm{O} \mathrm{III}] / \mathrm{H} \alpha$ flux ratios. The NW ear is only barely detected in [O III], while it brightens in $\mathrm{H} \alpha$ towards the edges of the $\mathrm{FoV}$, so that $[\mathrm{O}$ III] $] / \mathrm{H} \alpha \lesssim$ $1 / 10$. The northern ear, however, is clearly detected in [O III] with $[\mathrm{O} \mathrm{III}] / \mathrm{H} \alpha \approx 1 / 4$. These values are much lower compared to the central high-SB region. There (i.e., within the $1.25 \times 10^{-15} \mathrm{erg} \mathrm{s}^{-1} \mathrm{~cm}^{-2} \operatorname{arcsec}^{-2} \mathrm{H} \alpha \mathrm{SB}$ contour) we measure 


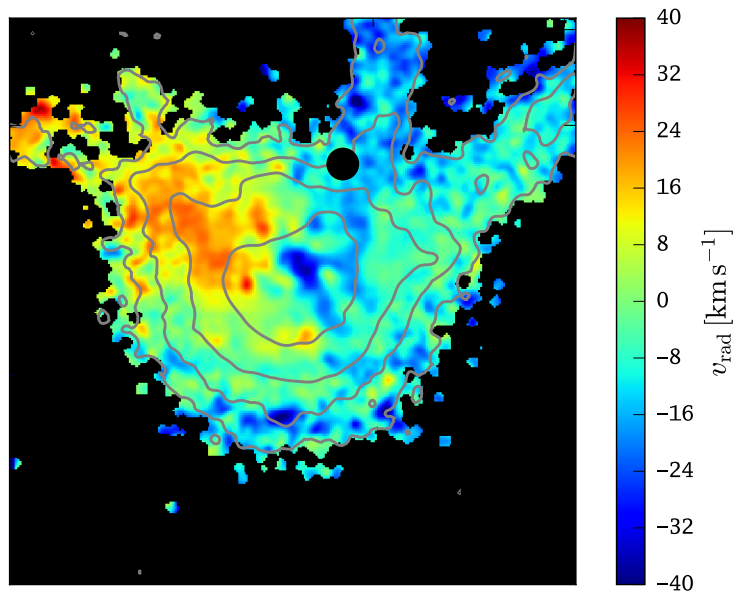

Fig. 3. $\mathrm{H} \alpha$ line of sight velocity field with $\mathrm{H} \alpha$ SB contours from Fig. 1. A background [O II] $\lambda 3727$ emitter at $z=0.78$ coincident with $\mathrm{H} \alpha$ contaminated the fit towards the north and hence the respective region is masked (black circle). We spatially smoothed the data cube with a top hat filter $\left(r=1^{\prime \prime}\right)$ to enhance the signal to noise for the fit

$[\mathrm{O} \mathrm{III}] / \mathrm{H} \alpha=0.99$ and $[\mathrm{O} \mathrm{III}] / \mathrm{H} \beta=2.97$. The $[\mathrm{O} \mathrm{III}] / \mathrm{H} \beta$ (or $[\mathrm{O} \mathrm{III}] / \mathrm{H} \alpha$ ) ratio depends on metallicity and on the ionisation parameter (i.e. the ratio of ionising photon density to gas density). The lower ratio found in the ears could be due to lower density and/or lower metallicity in the CGM. Unfortunately, other than only marginal detections in [O III] $\lambda 4959$ and $\mathrm{H} \beta$, we do not find other nebular lines from the filaments in our data. Hence, we cannot make strong inferences on the physical conditions in the ears. Even $\mathrm{H} \beta$ is not detected significantly enough to permit the calculation of $\mathrm{H} \alpha / \mathrm{H} \beta$ ratios in those low-SB regions that would be needed to assess the presence of dust.

Compared to its main stellar body, SBS 0335-052E also exhibits significantly extended He II $\lambda 4686$ emission (Fig. 2). The distinct peaks in the high-SB He II image are co-spatial with the more evolved SSCs, indicating that here the He II emission is related to post-main sequence stars (Wolf-Rayet stars) and/or their remnants (as first obtained by Izotov et al. 2006). Moreover, towards the NW the He II emission is more elongated with a brightening on the rim of the shell. This points towards a locally enhanced UV radiation field caused by the shell shockfront (Izotov et al. 2001; Thuan \& Izotov 2005). The total He II flux, obtained by integrating over all spaxels within the $2.5 \times 10^{-18} \mathrm{erg} \mathrm{s}^{-1} \mathrm{~cm}^{-2} \operatorname{arcsec}^{-2} \mathrm{SB}$ contour, is $F_{\text {HeII }}=$ $(2.17 \pm 0.03) \times 10^{-15} \mathrm{erg} \mathrm{s}^{-1} \mathrm{~cm}^{-2}$. Other possible sources for producing He II ionising $E_{\gamma}>4$ Ry photons are very massive metalpoor O-stars or high-mass X-ray binaries (Thuan et al. 2004; Prestwich et al. 2013)

We investigate the kinematics of the ionised gas by fitting a 1D Gaussian profile to the $\mathrm{H} \alpha$ line in each spaxel. The resulting line-of-sight velocity field is shown in Fig. 3. Within the high-SB region our velocity field agrees with the results of Moiseev et al. (2015) who traced $\mathrm{H} \alpha$ emission to $\sim 10^{-17} \mathrm{erg} \mathrm{s}^{-1} \mathrm{~cm}^{-2} \operatorname{arcsec}^{-2}$. These authors interpreted the central blue-shifted arc-like features as kinematic disturbances caused by the expansion of the super-shell. The shell structure also manifests itself in a locally double peaked $\mathrm{H} \alpha$ profile, which is however only resolved in the VLT/GIRAFFE data from Izotov et al. (2006) that was taken at $\sim 5 \times$ higher spectral resolution compared to MUSE. The newly detected filaments connect seamlessly in velocity space to the central velocity field. Especially, the ear towards the north shows the same blueshift as the shell. The extended kinematical imprint of the shell in the filaments argues that these filaments are created by feedback from the starburst. To quantify the ionised gas kinematics we calculate the shearing velocity $v_{\text {shear }}$ and the intrinsic velocity dispersion ${ }^{1} \sigma_{0}$ (see review by Glazebrook 2013). Following the procedures detailed in Herenz et al. (2016), we obtain $v_{\text {shear }}=19.8 \mathrm{~km} \mathrm{~s}^{-1}$ and $\sigma_{0}=$ $29.2 \mathrm{~km} \mathrm{~s}^{-1}$. Our derived $\sigma_{0}$ is in excellent agreement with the measurement by Moiseev et al. (2015): $\sigma_{0}=30.6 \pm 1.6 \mathrm{~km} \mathrm{~s}^{-1}$. As $v_{\text {shear }} / \sigma_{0}=0.68<1$, SBS 0335-052E qualifies as a dispersion dominated system. Dispersion dominated kinematics are commonly found among high-redshift star-forming galaxies (e.g. Turner et al. 2017). Herenz et al. (2016) showed that $v_{\text {shear }} / \sigma_{0}<1$ appears to be a necessary, but not solely sufficient criterion for galaxies to have Lyman $\alpha$ photons escaping. The escape of Lyman $\alpha$ photons can be theoretically linked to the leakage of LyC emission (Verhamme et al. 2015, for recent observational evidence see Verhamme et al. 2017). However, towards our line of sight Ly $\alpha$ is strongly absorbed (Thuan \& Izotov 1997; James et al. 2014), and there are only tentative signatures of a possible extended low-SB Ly $\alpha$ halo (Östlin et al. 2009). However, as we argue in Sect. 4, we believe that the low neutral column along the filaments facilitate $\mathrm{LyC}$ (and possibly $\mathrm{Ly} \alpha$ ) escape in this galaxy.

Lastly, we also point out that the galaxy is embedded in diffuse extended $\mathrm{H} \alpha$ emission. Detections of diffuse ionised halo gas far away from the main stellar body have been presented for individual spiral galaxies (e.g. Hlavacek-Larrondo et al. 2011) and in stacks of SDSS spectra (Zhang et al. 2016). Measurements of this diffuse component could provide constraints on the multi-phase nature of the CGM. We claim a significant detection out to $15^{\prime \prime}(4.1 \mathrm{kpc})$ from the main stellar body at $\sim 10^{-18} \mathrm{erg} \mathrm{s}^{-1} \mathrm{~cm}^{-2} \operatorname{arcsec}^{-2}$ (lowest iso-SB contour in Fig. 1). Constructing a SB profile without including the regions of the ears, we even measure diffuse emission out to $30^{\prime \prime}$ at $\gtrsim 10^{-19} \mathrm{erg} \mathrm{s}^{-1} \mathrm{~cm}^{-2} \operatorname{arcsec}^{-2}$. However, given the compactness of the high-SB region $-95 \%$ of the total encircled energy are within $2^{\prime \prime}$ - and that the SB-profile spans more than six orders of magnitude within $30^{\prime \prime}$, diffuse scattered light from telescope optics and/or the atmosphere cannot be neglected as a cause for the observed halo (Sandin 2014). As detailed by Sandin (2014) an accurate determination of the MUSE point spread function out to radii $\gtrsim 30^{\prime \prime}$ would be needed to accurately account for this effect, but such a measurement is not available.

\section{Discussion and conclusions}

Star formation in SBS 0335-052E is concentrated in six SSCs (Thuan et al. 1997) that have ages $\lesssim 10 \mathrm{Myr}$ and masses $\sim 5 \times 10^{5}-10^{6} M_{\odot}$. The two oldest ( 7-10 Myr), most massive $\left(\sim 10-20 \times 10^{5} M_{\odot}\right)$ SSCs are in the north and the two youngest ( $\lesssim 3 \mathrm{Myr}$ ), less massive ones $\left(\sim 4-5 \times 10^{5} M_{\odot}\right)$ are in the south (Thompson et al. 2009; Adamo et al. 2010). The starburst might have been triggered either by the recent encounter with its western companion or by a weak tidal interaction with the massive spiral NGC $1376\left(M \sim 10^{12} M_{\odot}\right)$, which is found at a projected distance of $150 \mathrm{kpc}$ (Pustilnik et al. 2001). The SSCs show an age sequence within $490 \mathrm{pc}\left(1.9^{\prime \prime}\right)$ from NW to south-east (SE). The age gradient points towards a scenario in which feedback from the oldest cluster in the NW ignited subsequent SF in the $\mathrm{SE}$ regions. This picture of unidirectional propagating $\mathrm{SF}$ as the main driver of the buildup of SBS 0335-052E is strengthened by an age gradient in faint stellar clusters detected with an

\footnotetext{
1 We correct for instrumental broadening using the wavelength dependent width of the MUSE line spread function given in Fig. 5 of Husser et al. (2016).
} 
unsharp-masking technique out to $\gtrsim 1 \mathrm{kpc} N E$ of the brightest SSC (Papaderos et al. 1998).

In the opposite direction of SF propagation, i.e. towards the lower density regions in the NW, feedback from the starburst is responsible for the super-shell structure. Initially the surface of such shells are very dense and hence optically thick for ionising photons. However, with continued mechanical energy input from SF the shells get accelerated and because of the density contrast with the ambient halo gas, Rayleigh-Taylor instabilities form. These fragment the surface of the shell and thus open funnels through which ionising photons start leaking into the halo. The precise timeline of bubble evolution depends sensitively on the density distribution of the gas and the mechanical energy input from the starburst (Fujita et al. 2003), but since we observe the ionised filaments the shell must be past this so-called "blowout". Ionising photons are now able to leak through the "cracks" of the shell into the CGM of much lower density, thereby creating the observed ionised filaments. Moreover, the hot wind that pushes through the cracks also contributes to ionising the ambient medium via shocks (Cooper et al. 2008).

Further inferences can be made when comparing our MUSE observations to inteferometric $21 \mathrm{~cm}$ imaging of the system. The highest spatial resolution H I images ( $\sim 4^{\prime \prime}$ beam) by Ekta et al. (2009) show indeed that the highest column densities $\left(N_{\mathrm{HI}} \sim\right.$ $10^{21} \mathrm{~cm}^{-2}$ ) are confined to the south-east, i.e. opposite from the direction of the expanding shell. With increased sensitivity at lower spatial resolution ( $\sim 7^{\prime \prime}$ beam), H I at lower column density $\left(\sim 10^{20} \mathrm{~cm}^{-2}\right)$ aligned with the direction of the shell becomes visible. Both these observational facts are consistent with the invoked scenario of feedback driven SF propagation in the highdensity SE regions and the feedback driven compression of neutral gas at the shell front towards the regions of lower density in the NE. At even lower resolution and higher sensitivity the images by Ekta et al. (2009) reveal a tidal feature slightly west of the NW ionised filament with $N_{\mathrm{HI}} \approx 7 \times 10^{19} \mathrm{~cm}^{-2}$. While the spatial resolution at this sensitivity appears insufficient to infer a direct spatial connection between filaments and neutral halo gas, it still shows that the bulk of $N_{\mathrm{HI}} \gtrsim 10^{19} \mathrm{~cm}^{-2}$ gas must be within the MUSE FoV, and that both the N and NW filament extend into regions of lower neutral columns. Thus, while we do not cover the full extend of the filaments, they appear to have penetrated completely through the bulk of the neutral gaseous halo of the system. Consequently, the reduced neutral fraction within those ionised filaments could advocate an escape of LyC photons from the starburst into the intergalactic medium.

To conclude, we have presented arguments for the ionised filaments of SBS 0335-052E towards the N and NW that are caused by SF-driven feedback, while feedback driven SF propagation proceeds towards the opposite direction in this galaxy. Interestingly, a large percentage of extremely metal-deficient starburst galaxies show cometary morphologies. This is regarded as evidence that unidirectional SF propagation is common in such systems (Papaderos et al. 2008). Moreover, 7-10\% of high$z$ galaxies show similar morphologies (Straughn et al. 2006). In order to explain cometary morphologies (also known as "tadpole" galaxies) other mechanisms have also been put forth, namely early-stage mergers or stream-driven gas accretion from the cosmic-web (e.g. Rhoads et al. 2005; Sánchez Almeida et al. 2014; Straughn et al. 2015; Lagos et al. 2016). Nevertheless, we speculate that if propagating SF is indeed an important driver of dwarf galaxy formation at high redshift, a highly anistropic LyC escape into small solid angles would be expected. In this case only a small fraction of LyC leakers will be directly detectable. More importantly, accounting for this effect might be important when budgeting the ionising photon output from lowmass star-forming galaxies in order to assess their contribution for reionising the universe.

Acknowledgements. We thank P. M. Weilbacher for feedback on handling the sky-oversubtraction issue described in Sect. 2. M.H. acknowledges the support of the Swedish Research Council, Vetenskapsrådet, and the Swedish National Space Board (SNSB), and is Fellow of the Knut and Alice Wallenberg Foundation. This work was supported by Fundação para a Ciência e a Tecnologia (FCT) through national funds and by FEDER through COMPETE by the grants UID/FIS/04434/2013 \& POCI-01-0145-FEDER-007672 and PTDC/FISAST/3214/2012 \& FCOMP-01-0124-FEDER-029170. P.P. was supported by FCT through Investigador FCT contract IF/01220/2013/CP1191/CT0002.

\section{References}

Adamo, A., Zackrisson, E., Östlin, G., \& Hayes, M. 2010, ApJ, 725, 1620 Bacon, R., Accardo, M., Adjali, L., et al. 2010, in SPIE Conf. Ser., 7735 Bik, A., Östlin, G., Hayes, M., et al. 2015, A\&A, 576, L13

Cooper, J. L., Bicknell, G. V., Sutherland, R. S., \& Bland-Hawthorn, J. 2008, ApJ, 674, 157

Ekta, B., Pustilnik, S. A., \& Chengalur, J. N. 2009, MNRAS, 397, 963 Faisst, A. L. 2016, ApJ, 829, 99

Finley, H., Bouché, N., Contini, T., et al. 2017, A\&A, 605, A118

Fujita, A., Martin, C. L., Mac Low, M.-M., \& Abel, T. 2003, ApJ, 599, 50 Glazebrook, K. 2013, PASA, 30, 56

Guseva, N. G., Izotov, Y. I., Fricke, K. J., \& Henkel, C. 2017, A\&A, 599, A65 Hayes, M. 2015, PASA, 32, 27

Herenz, E. C., \& Wisotzki, L. 2017, A\&A, 602, A111

Herenz, E. C., Gruyters, P., Orlitova, I., et al. 2016, A\&A, 587, A78

Hlavacek-Larrondo, J., Marcelin, M., Epinat, B., et al. 2011, MNRAS, 416, 509 Husser, T.-O., Kamann, S., Dreizler, S., et al. 2016, A\&A, 588, A148

Izotov, I. I., Guseva, N. G., Lipovetskii, V. A., Kniazev, A. I., \& Stepanian, J. A. 1990, Nature, 343, 238

Izotov, Y. I., Chaffee, F. H., \& Schaerer, D. 2001, A\&A, 378, L45

Izotov, Y. I., Schaerer, D., Blecha, A., et al. 2006, A\&A, 459, 71

Izotov, Y. I., Guseva, N. G., Fricke, K. J., \& Papaderos, P. 2009, A\&A, 503, 61

Izotov, Y. I., Thuan, T. X., Guseva, N. G., \& Liss, S. E. 2017, ArXiv e-prints [arXiv: 1709.00202]

James, B. L., Aloisi, A., Heckman, T., Sohn, S. T., \& Wolfe, M. A. 2014, ApJ, 795, 109

Lagos, P., Demarco, R., Papaderos, P., et al. 2016, MNRAS, 456, 1549 Moiseev, A. V., Tikhonov, A. V., \& Klypin, A. 2015, MNRAS, 449, 3568 Obreschkow, D., Heywood, I., \& Rawlings, S. 2011, ApJ, 743, 84

Östlin, G., Hayes, M., Kunth, D., et al. 2009, AJ, 138, 923

Papaderos, P., Izotov, Y. I., Fricke, K. J., Thuan, T. X., \& Guseva, N. G. 1998, A\&A, 338, 43

Papaderos, P., Guseva, N. G., Izotov, Y. I., \& Fricke, K. J. 2008, A\&A, 491, 113 Prestwich, A. H., Tsantaki, M., Zezas, A., et al. 2013, ApJ, 769, 92

Pustil'nik, S. A., Lipovetsky, V. A., Izotov, Y. I., et al. 1997, Astron. Lett., 23, 308

Pustilnik, S. A., Brinks, E., Thuan, T. X., Lipovetsky, V. A., \& Izotov, Y. I. 2001, AJ, 121, 1413

Rauch, M., Becker, G. D., \& Haehnelt, M. G. 2016, MNRAS, 455, 3991

Rhoads, J. E., Panagia, N., Windhorst, R. A., et al. 2005, ApJ, 621, 582

Rutkowski, M. J., Scarlata, C., Henry, A., et al. 2017, ApJ, 841, L27

Sánchez Almeida, J., Morales-Luis, A. B., Muñoz-Tuñón, C., et al. 2014, ApJ, 783,45

Sandin, C. 2014, A\&A, 567, A97

Schaerer, D., Izotov, Y. I. Verhamme, A, et al. 2016, A\&A, 591, L8

Straughn, A. N., Cohen, S. H., Ryan, R. E., et al. 2006, ApJ, 639, 724

Straughn, A. N., Voyer, E. N., Eufrasio, R. T., et al. 2015, ApJ, 814, 97

Thompson, R. I., Sauvage, M., Kennicutt, R. C., et al. 2009, ApJ, 691, 1068

Thuan, T. X., \& Izotov, Y. I. 1997, ApJ, 489, 623

Thuan, T. X., \& Izotov, Y. I. 2005, ApJS, 161, 240

Thuan, T. X., Izotov, Y. I., \& Lipovetsky, V. A. 1997, ApJ, 477, 661

Thuan, T. X., Bauer, F. E., Papaderos, P., \& Izotov, Y. I. 2004, ApJ, 606, 213

Turner, O. J., Cirasuolo, M., Harrison, C. M., et al. 2017, MNRAS, 471, 1280

Verhamme, A., Orlitová, I., Schaerer, D., \& Hayes, M. 2015, A\&A, 578, A7

Verhamme, A., Orlitová, I., Schaerer, D., et al. 2017, A\&A, 597, A13

Weilbacher, P. M., Streicher, O., Urrutia, T., et al. 2014, in Astronomical Data Analysis Software and Systems XXIII, eds. N. Manset, \& P. Forshay, ASP Conf. Ser., 485, 451

Wisotzki, L., Bacon, R., Blaizot, J., et al. 2016, A\&A, 587, A98

Zhang, H., Zaritsky, D., Zhu, G., Ménard, B., \& Hogg, D. W. 2016, ApJ, 833, 276 